Dossier: V Simposio Internacional y IX Nacional sobre Derechos de la Niñez y la Adolescencia

\title{
La promoción de la lactancia materna y la alimentación del bebé en la comunidad: experiencia del proyecto PROLAMANCO
}

\author{
Lilliam Marín Arias \\ Universidad de Costa Rica, Costa Rica \\ lilliam.marin@ucr.ac.cr \\ https://orcid.org/0000-0001-9426-1225
}

Recibido: 1 de julio de 2020

Aceptado: 31 de setiembre de 2020

Resumen: Una adecuada nutrición y alimentación durante la infancia y niñez temprana es esencial para asegurar que los niños alcancen todo su potencial en relación al crecimiento, salud y desarrollo. A pesar de la evidencia científica que señala la importancia de la lactancia materna para el bebé, la madre, familia y sociedad, esta forma de alimentación es poco incentivada en Costa Rica. Desde el año 2011, el Instituto de Investigaciones en Salud (INISA) de la Universidad de Costa Rica desarrolla el proyecto de extensión docente "Promoción de la lactancia materna y la alimentación del niño y la niña menor de 1 año en la comunidad" (ED2842 Proyecto PROLAMANCO), como respuesta a la necesidad de Educación para la Salud en el tema de lactancia materna y alimentación del niño durante el primer año de vida que presentan las madres y familiares de niños pequeños, así como profesionales relacionados con la atención de los niños en el ámbito comunitario. En este trabajo se presentan algunos resultados del proyecto PROLAMANCO.

Palabras claves: Niñez; alimentación; lactancia materna; educación para la salud; política pública; salud

The promotion of breastfeeding and infant nutrition in the community: experience of the PROLAMANCO project

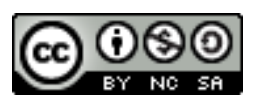

La Revista Estudios es editada por la Universidad de Costa Rica y se distribuye bajo una Licencia Creative Commons Atribución-NoComercial-CompartirIgual 3.0 Costa Rica. Para más información envíe un mensaje a revistaestudios.eeg@ucr.ac.cr. 
Dossier: V Simposio Internacional y IX Nacional sobre Derechos de la Niñez y la Adolescencia

\begin{abstract}
An adequate nutrition and feeding during infancy and early childhood is essential for assuring that children reach their full potential in relation to growth, health and development. Despite the scientific evidence that highlights the importance of breastfeeding for the baby, the mother, family, and society, this form of feeding is poorly incentivized in Costa Rica. Since 2011, The Research Institute of Health (INISA) of the University of Costa Rica developed the teaching extension project, "Promotion of breastfeeding and nutrition of children under 1 year in the community" (ED-2842 PROLAMANCO Project). Moreover, it was created as a response to the necessity of health education in the subject matter of breastfeeding and nutrition for children during their first year of life presented by the mothers, family, and professionals related to the care of children in the community. Some results of the PROLAMANCO project are presented.
\end{abstract}

Keywords: Childhood; nutrition; breastfeeding; health education; public policy; health

\title{
INTRODUCCIÓN
}

La alimentación y la nutrición humana continúan siendo factores determinantes del desarrollo y progreso de las sociedades (Martínez Rincón y Rodríguez Cisneros, 2002; Instituto de Nutrición de Centroamérica y Panamá, 2017). Una adecuada alimentación es esencial para asegurar que los niños alcancen una salud óptima, un desarrollo adecuado, un crecimiento armónico, un mayor bienestar y una elevada calidad de vida.

No obstante, el informe Estado de la Seguridad Alimentaria y la Nutrición en el Mundo 2019, señala que en el mundo existen más 820 millones de personas aproximadamente una de cada nueve- que padecieron hambre en 2018 (Organización de las Naciones Unidas para la Alimentación y la Agricultura, Fondo Internacional de Desarrollo Agrícola, Fondo de Naciones Unidas para la Infancia, Programa Mundial de Alimentos y Organización Mundial de la Salud, 2019). De ellas, se estima que 40 millones de personas viven en la región de América Latina

\section{() 100}

La Revista Estudios es editada por la Universidad de Costa Rica y se distribuye bajo una Licencia Creative Commons Atribución-NoComercial-CompartirIgual 3.0 Costa Rica. Para más información envíe un mensaje a 
Dossier: V Simposio Internacional y IX Nacional sobre Derechos de la Niñez y la Adolescencia y el Caribe, esto es, el 6,1\% de la población (Organización de las Naciones Unidas para la Alimentación y la Agricultura, Organización Panamericana de la Salud, Programa Mundial de Alimentos, Fondo de Naciones Unidas para la Infancia, 2018).

Además, en América Latina y el Caribe, la desnutrición infantil aguda está presente en 700000 niños y niñas menores de cinco años, lo que equivale a uno de cada 100. Asimismo, en esta misma región el retraso en el crecimiento (desnutrición crónica) afecta a 5,1 millones de los menores de cinco años (Organización de las Naciones Unidas para la Alimentación y la Agricultura, Fondo Internacional de Desarrollo Agrícola, Fondo de Naciones Unidas para la Infancia, Programa Mundial de Alimentos y Organización Mundial de la Salud, 2019). Por otra parte, alrededor de 3,9 millones de niños y niñas menores de 5 años en América Latina y el Caribe presenta sobrepeso infantil, lo que equivale al el 7,3\% de la población menor de 5 años, superando el promedio mundial que se encuentra en 5,6\% (Organización de las Naciones Unidas para la Alimentación y la Agricultura, Organización Panamericana de la Salud, Programa Mundial de Alimentos, Fondo de Naciones Unidas para la Infancia, 2018).

Según el documento titulado Impacto social y económico de la malnutrición: modelo de análisis y estudio piloto en Chile, el Ecuador y México (Fernández, Martínez, Carrasco y Palma, 2017), señala que la malnutrición en sus distintas manifestaciones (déficit o exceso) tiene consecuencias importantes en las familias y la sociedad, que abarcan la salud, con relación directa en morbilidades y mortalidad; el desarrollo de capacidades y los resultados educativos; la inclusión social y laboral; y el medioambiente. Todas estas consecuencias tienen a su vez impacto asociado a las pérdidas de productividad potencial respecto a la población que no está afectada por la malnutrición. Por todo ello, teniendo en cuenta sus 
Dossier: V Simposio Internacional y IX Nacional sobre Derechos de la Niñez y la Adolescencia

efectos y sus consecuencias negativas en la población a corto y largo plazo, es imprescindible poner fin a todas las formas de malnutrición.

Los estudios muestran que los principales factores de riesgo nutricionales relacionados con la muerte durante la niñez son la desnutrición aguda, la desnutrición crónica y el retardo del crecimiento intrauterino. Ante esta situación, los organismos internacionales de salud recomiendan que la lucha contra la desnutrición infantil inicie desde la etapa prenatal, esto para fomentar un adecuado control de salud y alimentación en las madres embarazadas y para continuar con una alimentación óptima durante la infancia y niñez temprana.

\section{Estrategia Mundial para la alimentación del lactante y Niño pequeño}

La Organización Mundial de la Salud (OMS) señala que una adecuada nutrición durante la infancia y niñez temprana es esencial para asegurar que los niños alcancen todo su potencial en relación al crecimiento, salud y desarrollo (Organización Mundial de la Salud, 2018). Una nutrición deficiente puede provocar un desgaste del estado nutricional y un debilitamiento del sistema inmunológico, lo que aumenta el riesgo de contraer enfermedades y obstaculiza la recuperación del cuerpo.

En el caso de la población infantil, existen una serie de elementos (atención de salud no adecuada, la aparición de infecciones, la reducida ingesta de alimentos, entre otros) que generan un círculo vicioso de la desnutrición que puede acabar con la vida de los niños. Adicional a estos elementos existen una serie de causas básicas (factores sociales, económicos y políticos, la desigualdad o la falta de educación) que favorecen la presencia de la inseguridad alimentaria y nutricional, lo que propicia al deterioro de la calidad de vida, la inequidad y la pobreza de importantes grupos de la población (Instituto de Nutrición de Centroamérica y Panamá, 2004).

\section{(C) $(\Theta \odot$}

La Revista Estudios es editada por la Universidad de Costa Rica y se distribuye bajo una Licencia Creative Commons Atribución-NoComercial-CompartirIgual 3.0 Costa Rica. Para más información envíe un mensaje a 
Dossier: V Simposio Internacional y IX Nacional sobre Derechos de la Niñez y la Adolescencia Los efectos más dramáticos de la malnutrición se observan en los lactantes y los niños más pequeños, quienes presentan la mayor morbilidad y mayor riesgo de muerte y discapacidad asociados a esta condición de salud (Organización Mundial de la Salud, 2003a). En este sentido, la OMS señala que en el año 2018 la desnutrición provocó 2,7 millones de muertes en niños, lo cual representa el 45\% de todas las muertes de niños. (Organización Mundial de la Salud, 2018).

Si bien, la Organización Panamericana de la Salud, de ahora en adelante OPS señala que en los últimos años la mayoría de países de América Latina y el Caribe han reducido sus tasas de mortalidad neonatal y en niños y niñas menores de 5 años, persisten grandes brechas entre los países, situación que debe ser trabajada a fondo (Organización Panamericana de la Salud, 2017). Por lo tanto, la alimentación del lactante y del niño pequeño es fundamental para mejorar la supervivencia infantil y fomentar un crecimiento y desarrollo saludables, siendo los dos primeros años de vida del niño especialmente importantes, puesto que la nutrición óptima durante este periodo reduce la morbilidad y la mortalidad, así como el riesgo de enfermedades crónicas en la etapa adulta, y mejora el desarrollo general de la población (Organización Mundial de la Salud, 2018).

Para dar respuesta a la situación de malnutrición en el mundo durante los primeros años de vida, la Organización Mundial de la Salud (OMS) y el Fondo de Naciones Unidas para la Infancia (UNICEF) formularon en el año 2002 la Estrategia mundial para la alimentación del lactante y el niño pequeño (Organización Mundial de la Salud, 2003b). La estrategia fue desarrollada con el propósito de revitalizar las medidas para fomentar, proteger y apoyar una alimentación adecuada en los primeros años de vida de los niños (Organización Mundial de la Salud, 2003b) y tiene como fundamento la Declaración de Innocenti (Fondo de las Naciones Unidas para la Infancia, s.f.) y la Iniciativa hospitales amigos de los niños (World Health Organization y Wellstar International, 1999).

\section{(c) (i) (-)}

La Revista Estudios es editada por la Universidad de Costa Rica y se distribuye bajo una Licencia Creative Commons Atribución-NoComercial-CompartirIgual 3.0 Costa Rica. Para más información envíe un mensaje a 
Dossier: V Simposio Internacional y IX Nacional sobre Derechos de la Niñez y la Adolescencia

Las recomendaciones que la Estrategia mundial señala para garantizar una alimentación del lactante y el niño pequeño óptima, son las siguientes:

- Lactancia materna exclusiva durante los primeros 6 meses de vida (180 días), pues permite proporcionar un alimento ideal para el crecimiento y el desarrollo sano. Además, es parte integrante del proceso reproductivo, con repercusiones importantes en la salud de las madres y en el desarrollo de la sociedad (Organización Mundial de la Salud, 2003b).

- A partir de los 6 meses de edad, los lactantes deben iniciar la alimentación complementaria, adecuada y segura, manteniendo la lactancia materna hasta los dos años de edad o más.

En ésta se hace un llamado para que el tema de la alimentación en los primeros años de vida no sólo sea responsabilidad de los gobiernos sino también de las organizaciones internacionales, organizaciones no gubernamentales y otras instancias involucradas en el ámbito comunal que trabajen en la promoción de la salud de la población (Cuadro 1) (Organización Mundial de la Salud, s.f.)

\section{(c) (i) (2)}

La Revista Estudios es editada por la Universidad de Costa Rica y se distribuye bajo una Licencia Creative Commons Atribución-NoComercial-CompartirIgual 3.0 Costa Rica. Para más información envíe un mensaje a revistaestudios.eeg@ucr.ac.cr. 
Dossier: V Simposio Internacional y IX Nacional sobre Derechos de la Niñez y la Adolescencia

Cuadro 1. Áreas de acción en la Estrategia mundial para la alimentación del lactante y el niño pequeño

\begin{tabular}{ll}
\hline Gobiernos & Formular y aplicar una política integral sobre \\
& alimentación del lactante y del niño pequeño, en el \\
contexto de las políticas nacionales de nutrición, salud \\
infantil y reproductiva, y reducción de la pobreza. \\
- Supervisar la aplicación nacional del Código \\
Internacional de Comercialización de Sucedáneos de la \\
Leche Materna. \\
Promulgar leyes o medidas adicionales para proteger a \\
las familias de las influencias comerciales negativas en \\
el tema de alimentación. \\
Promulgar leyes para proteger el derecho a la lactancia \\
materna de las mujeres trabajadoras de conformidad \\
con las normas laborales internacionales.
\end{tabular}

Profesionales de

la salud
Todos los profesionales del área de la salud deben estar capacitados para proporcionar asesoramiento eficaz sobre la alimentación a las madres y familias. Sus servicios deberían extenderse a la comunidad.
Todas las madres y familias deberían tener:

- Acceso a un apoyo especializado para iniciar y mantener la lactancia exclusivamente materna durante 6 meses.

- Recibir apoyo especializado para introducir en la dieta del niño alimentos complementarios adecuados e inocuos en el momento oportuno.

- Apoyo para lograr mantener la lactancia materna hasta los dos años de edad o más.

\section{Fuente: Elaboración propia}

\section{(@) $\odot \Theta \odot$}

La Revista Estudios es editada por la Universidad de Costa Rica y se distribuye bajo una Licencia Creative Commons Atribución-NoComercial-CompartirIgual 3.0 Costa Rica. Para más información envíe un mensaje a 
Dossier: V Simposio Internacional y IX Nacional sobre Derechos de la Niñez y la Adolescencia

La lactancia materna es la forma ideal de alimentación para los lactantes, pues no solo brinda nutrientes, sino también defensas que los protege de enfermedades, lo cual favorece un crecimiento y desarrollo saludable. Incentivar el hábito de la lactancia materna mediante el cumplimiento de las recomendaciones de la OMS puede salvar la vida de más de 820000 niños por año, ya que esta práctica protege contra las enfermedades diarreicas e infecciones respiratorias. Asimismo, esta forma de alimentación disminuye los riesgos de cáncer de mama y de ovario en las mujeres que amamantan (Victora et al., 2016).

A nivel mundial, una minoría de lactantes y niños cumplen con las recomendaciones establecidas en la Estrategia mundial para la alimentación del lactante y el niño pequeño, pues, solo el $42 \%$ de los lactantes inician la lactancia materna dentro de la primera hora después del nacimiento (solo dos de cada cinco niños) (Fondo de las Naciones Unidas para la Infancia y Organización Mundial de la Salud, 2018) y el $43 \%$ de los bebés llegan a los 6 meses de la edad alimentados sólo con leche materna. A los 2 años de edad, el 45\% de los niños están siendo todavía alimentados con leche materna (United Nations Children's Fund, 2016)

\section{Normativa costarricense sobre lactancia materna y alimentación de los niños}

Como parte de los compromisos adquiridos en instancias internacionales, Costa Rica se ha comprometido a promover y proteger la lactancia materna y la alimentación de los niños tanto desde el ámbito normativo como del desarrollo de programas estatales de promoción de la lactancia materna y alimentación de los niños. En el país existen diferentes leyes que ofrecen una amplia cobertura a la promoción, protección de la salud y a la alimentación en los primeros años de vida, algunas de ellas hacen énfasis en la lactancia materna (Cuadro 2).

\section{(c) (i) (2)}

La Revista Estudios es editada por la Universidad de Costa Rica y se distribuye bajo una Licencia Creative Commons Atribución-NoComercial-CompartirIgual 3.0 Costa Rica. Para más información envíe un mensaje a 
Dossier: V Simposio Internacional y IX Nacional sobre Derechos de la Niñez y la Adolescencia

Cuadro 2. Marco normativo costarricense entorno al tema de lactancia materna

\begin{tabular}{|l|l|}
\hline \multicolumn{1}{|c|}{ Norma } & \multicolumn{1}{|c|}{ Fecha de aprobación } \\
\hline Código de Trabajo & $\begin{array}{l}\text { Congreso Constitucional de la } \\
\text { República de Costa Rica,1943 }\end{array}$ \\
\hline Constitución Política de Costa Rica & $\begin{array}{l}\text { Asamblea Nacional Constituyente, } \\
1949\end{array}$ \\
\hline Ley General de Salud & $\begin{array}{l}\text { Asamblea Legislativa de Costa Rica, } \\
1973\end{array}$ \\
\hline $\begin{array}{l}\text { Ley General de la Administración } \\
\text { Pública }\end{array}$ & $\begin{array}{l}\text { Asamblea Legislativa de Costa Rica, } \\
1978\end{array}$ \\
\hline $\begin{array}{l}\text { Ley Fomento de la Lactancia } \\
\text { Materna }\end{array}$ & $\begin{array}{l}\text { Asamblea Legislativa de Costa Rica, } \\
1994\end{array}$ \\
\hline $\begin{array}{l}\text { Código de la Niñez y de la } \\
\text { Adolescencia }\end{array}$ & $\begin{array}{l}\text { Asamblea Legislativa de Costa Rica, } \\
1998\end{array}$ \\
\hline $\begin{array}{l}\text { Política nacional de lactancia } \\
\text { materna }\end{array}$ & DM 8514-2008, 2008 \\
\hline $\begin{array}{l}\text { Reglamento de condiciones para las } \\
\text { salas de lactancia materna en los } \\
\text { centros de trabajo }\end{array}$ & Decreto №41080 MTSS-S, 2018 \\
\hline
\end{tabular}

Fuente: Elaboración propia

A pesar de contar con legislación y normativa en el tema, los datos actuales de lactancia materna en Costa Rica no son los mejores. De los niños y niñas que llegan a los 6 meses de edad, solo un 27,5\% recibieron lactancia materna exclusiva y un $39 \%$ recibieron lactancia materna predominante (Ministerio de Salud, Instituto Nacional de Estadística y Censos y Fondo de las Naciones Unidas para la Infancia, 2018). Esta situación pone en evidencia, que a pesar que la literatura científica señala la importancia de la lactancia materna para el bebé, la madre, familia y

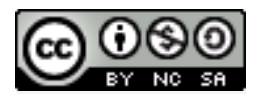

La Revista Estudios es editada por la Universidad de Costa Rica y se distribuye bajo una Licencia Creative Commons Atribución-NoComercial-CompartirIgual 3.0 Costa Rica. Para más información envíe un mensaje a revistaestudios.eeg@ucr.ac.cr. 
Dossier: V Simposio Internacional y IX Nacional sobre Derechos de la Niñez y la Adolescencia

sociedad, esta forma de alimentación es poco incentivada en nuestro país y socialmente se considera que la responsabilidad de esta práctica recae principalmente en la madre, por lo que se descuida la responsabilidad que tiene la familia, el Estado y la sociedad en fomentar y proteger la lactancia materna.

Como respuesta a la situación de lactancia materna, la Caja Costarricense del Seguro Social ha implementado en los hospitales nacionales y regionales del país las Clínicas de Lactancia Materna y Desarrollo. Actualmente, el país cuenta con 25 Clínicas distribuidas en diferentes zonas del país, las cuales ofrecen servicios de atención directa a la madre en proceso de amamantamiento, atención directa al recién nacido y su desarrollo, apoyo al proceso de extracción y conservación de la leche humana (Caja Costarricense del Seguro Social, 2012).

En el año 2009 el Ministerio de Salud de Costa Rica como ente rector de la salud del país, publica la Política Nacional de Lactancia Materna, con el objetivo de maximizar la salud integral de las madres, niñas y niños, jóvenes, familias y población en general. Esta política señala que "El amamantamiento es la norma biológica que debe orientar la alimentación del niño y de la niña, por lo que las instituciones públicas y privadas prestadoras de servicios a la niñez, deben garantizar las condiciones necesarias para que este grupo poblacional sea alimentado con lactancia materna, de manera exclusiva hasta los seis meses de edad y de forma complementaria hasta los dos años o más, con alimentos saludables, autóctonos y producidos en su comunidad" (Ministerio de Salud, 2009, p. 2).

Asimismo, la política menciona que las instituciones públicas y privadas de todos los sectores salud, educación, industria, economía, comercio, entre otros deben velar por el cumplimiento de la normativa vigente relacionada con las buenas prácticas de lactancia materna en todos los escenarios donde se tenga contacto con los niños, las niñas, sus madres y las familias. Asimismo, señala que las

La Revista Estudios es editada por la Universidad de Costa Rica y se distribuye bajo una Licencia Creative Commons Atribución-NoComercial-CompartirIgual 3.0 Costa Rica. Para más información envíe un mensaje a revistaestudios.eeg@ucr.ac.cr. 
Dossier: V Simposio Internacional y IX Nacional sobre Derechos de la Niñez y la Adolescencia instituciones deben apoyar, coordinar y realizar actividades alusivas a la celebración de la Semana Mundial de la Lactancia Materna, con el fin de promocionar en la población la alimentación al seno materno de manera exclusiva hasta los seis meses de edad y de forma complementaria hasta los dos años o más (Ministerio de Salud, 2009).

\section{Experiencia del proyecto PROLAMANCO}

En el año 2011, el Instituto de Investigaciones en Salud (INISA) inscribe ante la Vicerrectoría de Acción Social de la Universidad de Costa Rica el proyecto de extensión docente "Promoción de la lactancia materna y la alimentación del niño y la niña menor de 1 año en la comunidad" (ED-2842 Proyecto PROLAMANCO)

El proyecto PROLAMANCO nace como respuesta a la necesidad de Educación para la salud en el tema de lactancia materna y alimentación del niño durante el primer año de vida que presentan las madres y familiares de niños pequeños, así como profesionales relacionados con la atención de los niños en el ámbito comunitario.

Este vacío se hizo evidente en dos diferentes proyectos de investigación y acción social realizados por el INISA en comunidades del área urbana y rural de Costa Rica. Estos fueron el proyecto de extensión docente ED-2190 "Madres ayudando a madres", y el proyecto de investigación 742-A9-323 "Factores individuales y familiares que inciden en la práctica de la lactancia materna en un grupo de mujeres costarricenses residentes en una zona urbana y en una zona rural de Costa Rica". El contacto con las personas en su cotidianidad permitió conocer la realidad que viven las madres con niños pequeños, el nivel de conocimiento que, en el tema de alimentación durante el primer año de vida, tienen estas mujeres y sus familias, así como la inexacta orientación sobre el tema que reciben de los profesionales de salud.

\section{(c) (i) (-)}

La Revista Estudios es editada por la Universidad de Costa Rica y se distribuye bajo una Licencia Creative Commons Atribución-NoComercial-CompartirIgual 3.0 Costa Rica. Para más información envíe un mensaje a 
Dossier: V Simposio Internacional y IX Nacional sobre Derechos de la Niñez y la Adolescencia Los estudios realizados en Costa Rica sobre lactancia materna y alimentación de los niños, indican que las mujeres pueden amamantar y alimentar a sus hijos de manera exitosa, siempre y cuando dispongan de adecuada información, orientación y apoyo por parte de su familia, de su entorno y del sistema de atención de salud (Marín-Arias, Ureña Cascante y Fernández Rojas, 2017; Gutiérrez-Obregón y Marín-Arias, 2017).

Por esta situación, el proyecto PROLAMANCO busca poner al alcance de la población costarricense conocimiento actualizado en el tema de alimentación y desarrollo en los primeros años de vida, con el propósito contribuir a la salud, crecimiento y desarrollo de los niños y niñas menores de un año. Todo esto mediante la promoción, fomento y defensa de la lactancia materna, pero también a través de una adecuada introducción de los alimentos diferentes a la leche materna a partir de los seis meses de edad. Este propósito se desarrolla empleando la Educación para la salud, como parte de las herramientas indispensables dentro de la Promoción de la salud (de Vincezi y Tedesco, 2009, p.4). Los principales fundamentos conceptuales del proyecto PROLAMANCO son los siguientes:

A. Las iniciativas internacionales y nacionales en el tema de alimentación infantil, como los Objetivos de Desarrollo Sostenible (Organización de Naciones Unidas, 2018), la Estrategia Mundial para la Alimentación del Lactante y el Niño Pequeño, y la Política Nacional de Lactancia Materna.

B. Las recomendaciones nacionales e internacionales sobre alimentación en el primer año de vida, donde se resalta la importancia de la lactancia materna y la adecuada introducción de alimentos en los bebés.

C. La Educación para la salud, que comprende la alfabetización sanitaria, la mejora del conocimiento y la motivación de la población, así como el desarrollo de habilidades y la autoestima personal que conduzcan a la

\section{(c) (i) (2)}

La Revista Estudios es editada por la Universidad de Costa Rica y se distribuye bajo una Licencia Creative Commons Atribución-NoComercial-CompartirIgual 3.0 Costa Rica. Para más información envíe un mensaje a revistaestudios.eeg@ucr.ac.cr. 
Dossier: V Simposio Internacional y IX Nacional sobre Derechos de la Niñez y la Adolescencia adopción de medidas destinadas a la mejora de la salud individual y de la comunidad (Organización Mundial de la Salud, 1998).

D. La comunicación para la salud, que es una estrategia clave para informar a la población sobre aspectos de salud y para mantener cuestiones sanitarias importantes en la agenda pública. El uso de los medios informativos actuales para difundir información sobre salud entre la población, aumenta la concienciación sobre aspectos específicos de la salud individual y colectiva y sobre la importancia de la salud en el desarrollo (Organización Mundial de la Salud, 1998)

Para alcanzar sus propósitos, el proyecto PROLAMANCO desarrolla diferentes actividades orientadas no sólo hacia las madres y familias de niños pequeños, sino también hacia la población en general y hacia diversos grupos de profesionales que se relacionan con población materno infantil. El trabajo se distribuye en tres áreas de acción que articulan las actividades del proyecto (Cuadro 3).

\section{(c) (i) (-)}

La Revista Estudios es editada por la Universidad de Costa Rica y se distribuye bajo una Licencia Creative Commons Atribución-NoComercial-CompartirIgual 3.0 Costa Rica. Para más información envíe un mensaje a revistaestudios.eeg@ucr.ac.cr. 
Dossier: V Simposio Internacional y IX Nacional sobre Derechos de la Niñez y la Adolescencia

Cuadro 3. Áreas de acción del proyecto PROLAMANCO.

\begin{tabular}{|c|c|}
\hline Áreas de acción & Actividades realizadas \\
\hline $\begin{array}{l}\text { Propiciar espacios de discusión sobre la } \\
\text { importancia de la alimentación en los } \\
\text { primeros dos años de vida }\end{array}$ & $\begin{array}{l}\text { - Jornada de Promoción de Lactancia } \\
\text { Materna en el marco de la celebración de la } \\
\text { Semana Mundial de Lactancia Materna. Se } \\
\text { iniciaron en el año } 2014 \text { y se han realizado } \\
5 \text { jornadas. } \\
\text { - Curso "Crianza con apego" (2017), } \\
\text { impartido por el médico pediatra español } \\
\text { Dr. José María Aparicio. fundador de } \\
\text { APILAN y de la página de consulta e- } \\
\text { lactancia.org. } \\
\text { Ponencia "Pequeños grandes clientes: Los } \\
\text { bebés y las transnacionales de la } \\
\text { alimentación" (2018), impartida por el Dr. } \\
\text { Fernando Vallone, médico pediatra } \\
\text { argentino. }\end{array}$ \\
\hline $\begin{array}{l}\text { La promoción, fomento y protección de la } \\
\text { lactancia materna y alimentación } \\
\text { saludable de los niños en los primeros } \\
\text { dos años de vida en diversas instancias } \\
\text { en el ámbito comunitario }\end{array}$ & $\begin{array}{l}\text { - Participación en entrevistas de radio y } \\
\text { televisión sobre el tema de lactancia } \\
\text { materna y alimentación de los niños en los } \\
\text { primeros años de vida. } \\
\text { - Charlas sobre el tema de lactancia materna } \\
\text { y alimentación de los niños pequeños en } \\
\text { centros de salud u otras instancias. } \\
\text { - Charlas sobre lactancia materna e } \\
\text { introducción de alimentos en los bebés en } \\
\text { curso de preparación para el parto. } \\
\text { - Ponencias en sesiones médicas de centros } \\
\text { de salud. } \\
\text { Ponencias en talleres de actualización en } \\
\text { lactancia materna. } \\
\text { Asesorías telefónicas a madres que } \\
\text { requieren orientación inmediata sobre } \\
\text { aspectos varios en lactancia materna. } \\
\text { Asesorías a dependencias universitarias } \\
\text { sobre salas de lactancia materna, atención } \\
\text { a madre trabajadora en proceso de } \\
\text { lactancia materna. }\end{array}$ \\
\hline $\begin{array}{l}\text { Curso de Educación para la salud sobre } \\
\text { lactancia materna, introducción de } \\
\text { alimentos a partir de los seis meses de } \\
\text { vida y estimulación temprana }\end{array}$ & $\begin{array}{l}\text { - Cursos de Educación para la salud } \\
\text { orientados a público en general. } \\
\text { - Curso de actualización para profesionales. }\end{array}$ \\
\hline
\end{tabular}

Fuente: Elaboración propia

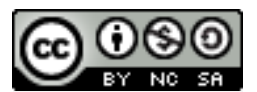

La Revista Estudios es editada por la Universidad de Costa Rica y se distribuye bajo una Licencia Creative Commons Atribución-NoComercial-CompartirIgual 3.0 Costa Rica. Para más información envíe un mensaje a 
Dossier: V Simposio Internacional y IX Nacional sobre Derechos de la Niñez y la Adolescencia

Con relación al curso de Educación para la Salud indicado en el Cuadro 3, se debe añadir que está organizado para impartirse en 6 sesiones, dirigido a madres y familias con niños pequeños, personas cuidadoras o profesionales en el ámbito comunitario que se relacionan con niños. Este curso busca la promoción y recuperación de la cultura de la lactancia materna en la sociedad costarricense.

En los últimos años, el curso de Educación para la salud sobre lactancia materna, introducción de alimentos a partir de los seis meses de vida y estimulación temprana ha tenido gran alcance en la población costarricense, convirtiéndose en una actividad relevante dentro del proyecto PROLAMANCO, siendo hasta el momento, el único curso que ofrece la Universidad de Costa Rica con estas características. Hasta julio del 2019 el proyecto ha impartido 27 cursos para el público general con una participación de 1145 personas, lo que representa una media superior a los 40 participantes por curso. De estos participantes, el $93 \%$ corresponden a mujeres y el porcentaje restante a hombres.

Asimismo, al curso asisten principalmente mujeres en calidad de futuras madres y con niños pequeños. El mismo, se imparte en la Sede Rodrigo Facio en San Pedro de Montes de Oca, no obstante, se ha impartido en otros lugares como Cartago (Centro y La Unión), Guanacaste (Liberia) y San José (Alajuelita). Es importante destacar, que en las ocasiones que se ha brindado en San José, han asistido personas de lugares lejanos a la sede Rodrigo Facio, como Santa María de Dota, Grecia, Poás, Puriscal, Esparza, Siquirres y Liberia (Figura 1), lo que demuestra la necesidad que en este tema tienen las personas de otras zonas del país.

\footnotetext{
(c) (1) @()

La Revista Estudios es editada por la Universidad de Costa Rica y se distribuye bajo una Licencia Creative Commons Atribución-NoComercial-CompartirIgual 3.0 Costa Rica. Para más información envíe un mensaje a revistaestudios.eeg@ucr.ac.cr.
} 
Dossier: V Simposio Internacional y IX Nacional sobre Derechos de la Niñez y la Adolescencia

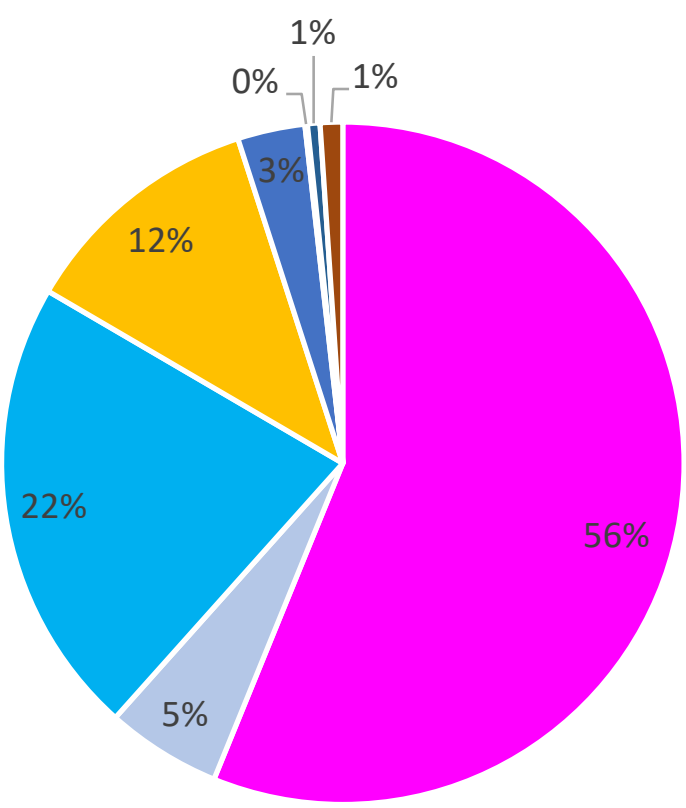

- San José
- Heredia
- Limón

- Alajuela

- Guanacaste

- No responde

Figura 1. Provincia de procedencia de las personas asistentes al curso de Educación para la salud sobre lactancia e introducción de alimentos en los niños.

Fuente: Elaboración propia

El curso es gratuito, abierto al público general y los asistentes pueden asistir con sus hijos (en caso de tenerlos). Si bien el curso se planeó para un público no especializado, se ha convertido en una opción para profesionales que se relacionan con niños pequeños (medicina, enfermería, nutrición, psicología, preescolar, terapia del lenguaje, entre otros), pues estos profesionales manifiestan la necesidad de contar con información sobre el tema (Figura 2). Esta situación ha propiciado que

\section{(c) $)(1)(2)$}

La Revista Estudios es editada por la Universidad de Costa Rica y se distribuye bajo una Licencia Creative Commons Atribución-NoComercial-CompartirIgual 3.0 Costa Rica. Para más información envíe un mensaje a revistaestudios.eeg@ucr.ac.cr. 
Dossier: V Simposio Internacional y IX Nacional sobre Derechos de la Niñez y la Adolescencia en cuatro ocasiones el proyecto también haya propiciado la realización de un curso de actualización profesional con el propósito ofrecer a los profesionales una revisión sobre el tema y la forma correcta de trabajar la alimentación en los primeros años de vida. Estos han tenido una participación de 116 personas y se han llevado a cabo en San José y Liberia.
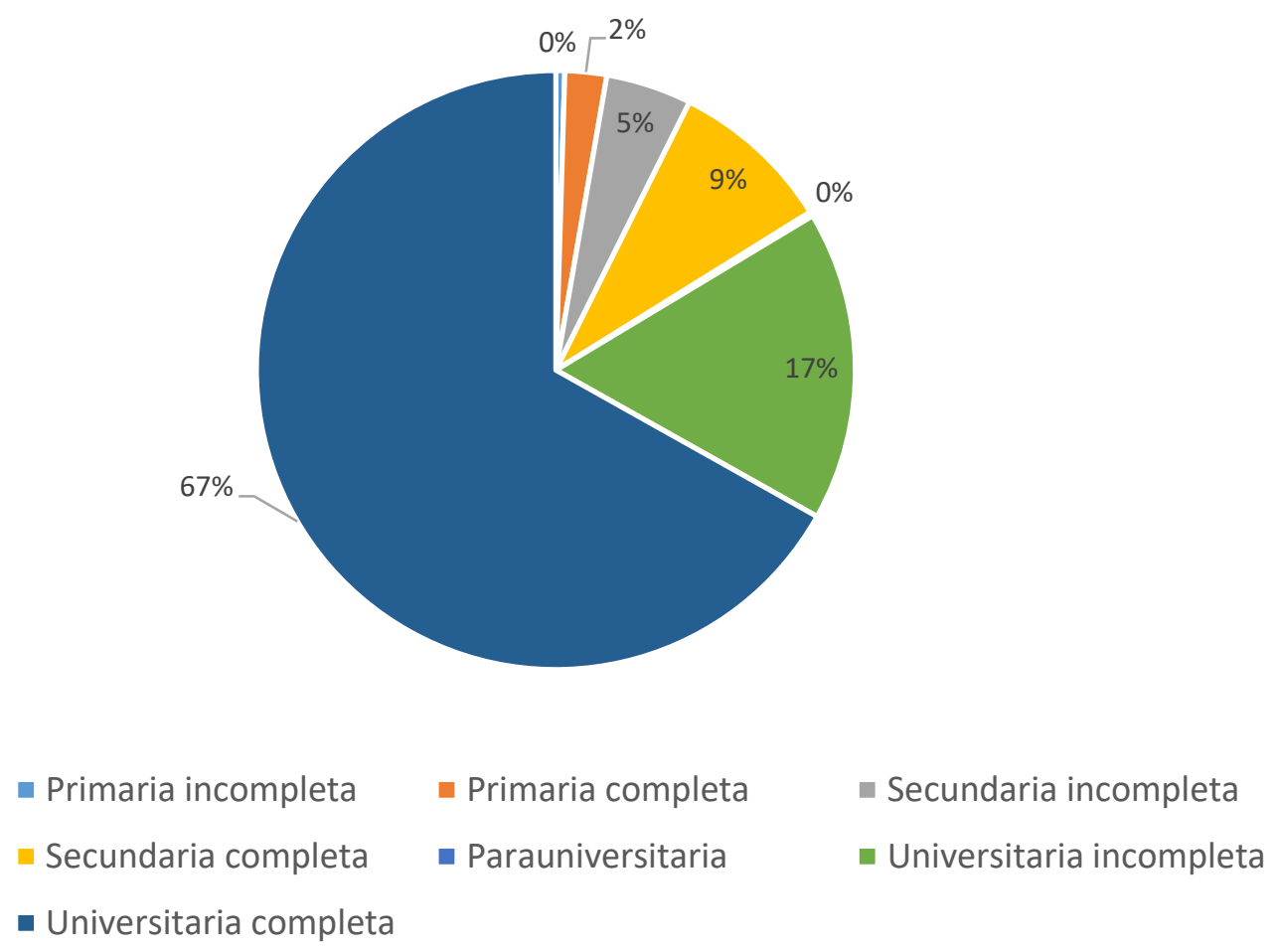

Figura 2. Escolaridad de las personas asistentes al curso de Educación para la salud sobre lactancia e introducción de alimentos en los niños.

Fuente: Elaboración propia

\section{(@) $(\Theta \odot$}

La Revista Estudios es editada por la Universidad de Costa Rica y se distribuye bajo una Licencia Creative Commons Atribución-NoComercial-CompartirIgual 3.0 Costa Rica. Para más información envíe un mensaje a revistaestudios.eeg@ucr.ac.cr. 
Dossier: V Simposio Internacional y IX Nacional sobre Derechos de la Niñez y la Adolescencia

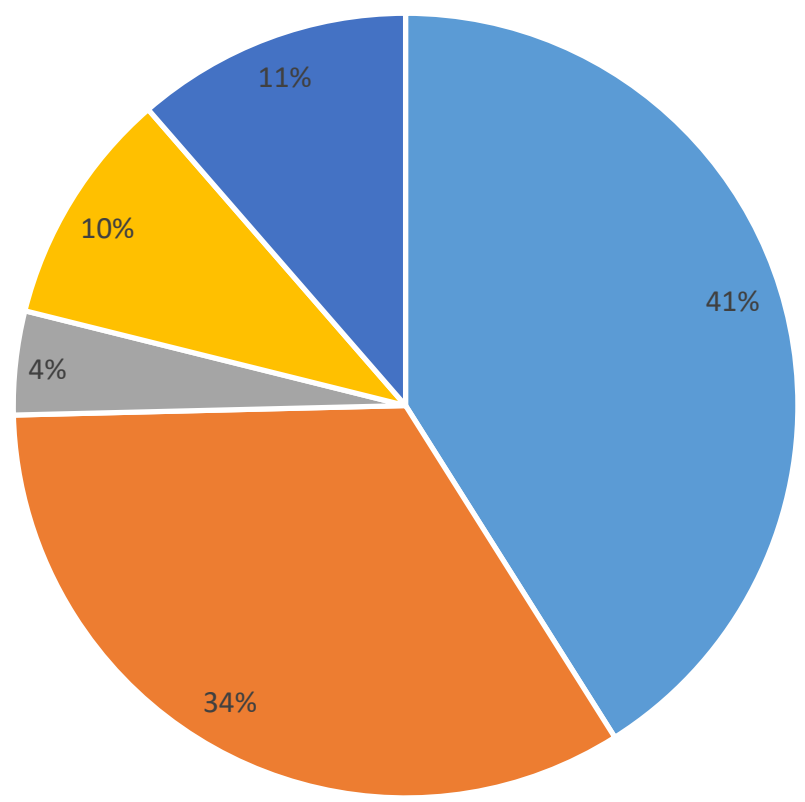

- Referencias de amistades y familiares - Redes sociales

घ Correo electrónico $\quad$ Medios universitarios

- Otros

Figura 3. Forma por el cual la persona participante se enteró del curso de Educación para la salud sobre lactancia e introducción de alimentos en los niños

Fuente: Elaboración propia

\section{Consideraciones finales}

Para asegurar que la práctica de lactancia materna sea una realidad en el ámbito comunitario y que los beneficios que ofrece esta práctica sean efectivos para el beneficio de la niñez en los primeros años de vida, se hace necesario incrementar la inversión en programas de apoyo en la comunidad y la acción política de las instituciones del Estado. A pesar de la existencia de una legislación en Costa Rica

\section{(C) $(\mathbb{Q} \Theta(-)$}

La Revista Estudios es editada por la Universidad de Costa Rica y se distribuye bajo una Licencia Creative Commons Atribución-NoComercial-CompartirIgual 3.0 Costa Rica. Para más información envíe un mensaje a revistaestudios.eeg@ucr.ac.cr. 
Dossier: V Simposio Internacional y IX Nacional sobre Derechos de la Niñez y la Adolescencia sobre la alimentación de los niños y niñas, la promoción de la lactancia materna es escasa debido a la falta de acciones de los sectores involucrados con la salud integral del niño, la niña, la madre y la familia.

Se hace necesario, por lo tanto, que la promoción y protección de la lactancia materna y la adecuada introducción de alimentos complementarios a partir de los seis meses de edad de los niños y niñas se considere una prioridad dentro de la agenda de trabajo de las instituciones del Estado involucradas con la salud integral de este grupo. Para ello, es necesario trabajar de forma coordinada entre las instituciones, relacionando las políticas nacionales y las acciones locales con miras a un mismo propósito: asegurar un adecuado estado nutricional y de salud en los primeros años de vida, de modo que se garantice una sana niñez y adolescencia, así como unos futuros adultos sanos.

El trabajo de las instituciones no debe quedarse en un discurso dentro los niveles jerárquicos superiores, sino convertirse en una práctica real y cotidiana dirigida a las personas más necesitadas de este proceso, es decir, a las madres y a las familias que viven el proceso de lactancia materna y alimentación de los niños y niñas en la comunidad. Los profesionales de la salud y afines (médicos, enfermeras, farmacéuticos, odontólogos, nutricionistas, psicólogos, trabajadores sociales, educadores de etapa preescolar, entre otros) deben cumplir una función primordial para promocionar y proteger la alimentación adecuada de los niños y niñas.

Sin embargo, la formación de los profesionales no se corresponde con esta necesidad. La investigación científica de estos temas, pone en evidencia la necesidad de revisar los contenidos de los planes curriculares de algunos profesionales. Entre otros aspectos, la formación académica debe relacionarse con las políticas nacionales y la realidad internacional en el tema, y así poder orientar adecuadamente a la población.

\section{(c) (i) (2)}

La Revista Estudios es editada por la Universidad de Costa Rica y se distribuye bajo una Licencia Creative Commons Atribución-NoComercial-CompartirIgual 3.0 Costa Rica. Para más información envíe un mensaje a 


\section{Dossier: V Simposio Internacional y IX Nacional sobre Derechos de la Niñez y la Adolescencia}

Se hace necesario que las universidades públicas y privadas, actualice los planes de estudios y ofrecer a los futuros profesionales del área de la salud las competencias necesarias, para abordar y orientar el tema de alimentación y desarrollo en la población costarricense.

Debido al compromiso social de las universidades púbicas para responder a las necesidades de formación y educación de la sociedad costarricense, ésta debería convertirse en un espacio idóneo para promover y defender la lactancia materna y la alimentación adecuada de los niños y niñas en los primeros años de vida.

\section{Bibliografía}

Caja Costarricense del Seguro Social. (2012). Manual de implementación de clínicas de lactancia materna y desarrollo. San José: Costa Rica. Recuperado de

https://www.ministeriodesalud.go.cr/gestores_en_salud/lactancia/CNLM_ma nual_lactac_materna_y_desarrollo_2012.pdf

De Vincezi, A. y Tedesco,F. (2009). La educación como proceso de mejoramiento de la calidad de vida de los individuos y de la comunidad. Revista Iberoamericana de Educación, 49 (7), 1-12. doi: 10.35362/rie4972047

Fernández, A., Martínez, R., Carrasco, I. y Palma, A. (2017). Impacto social y económico de la malnutrición: modelo de análisis y estudio piloto en Chile, el Ecuador $y$ México. Recuperado de https://repositorio.cepal.org/bitstream/handle/11362/42535/1/S1700443_es. pdf

\section{(c) (i) (9)}

La Revista Estudios es editada por la Universidad de Costa Rica y se distribuye bajo una Licencia Creative Commons Atribución-NoComercial-CompartirIgual 3.0 Costa Rica. Para más información envíe un mensaje a revistaestudios.eeg@ucr.ac.cr. 
Dossier: V Simposio Internacional y IX Nacional sobre Derechos de la Niñez y la Adolescencia

Fondo de Naciones Unidas para la Infancia [UNICEF]. (s.f ). Innocenti Declaration on the Protection, Promotion and Support of Breastfeeding. Recuperado de: https://www.unicef.org/spanish/nutrition/index_24807.html

Fondo de las Naciones Unidas para la Infancia [UNICEF]. (s.f.). Lactancia materna. Recuperado de https://www.unicef.org/spanish/nutrition/index_24824.html

Fondo de las Naciones Unidas para la Infancia [UNICEF] y Organización Mundial de la Salud [OMS]. (2018). Capturar el momento: Inicio temprano de la lactancia materna: El mejor comienzo para cada recién nacido. Nueva York: UNICEF. Recuperado de https://www.unicef.org/spanish/publications/files/UNICEF_WHO_Capture_th e_moment_EIBF_2018_Sp.pdf

Gutiérrez Obregón, Y. y Marín Arias, L. (2017). Significado de lactancia materna y leche materna para las madres de una comunidad urbana y otra rural de Costa Rica. Población y Salud en Mesoamérica. 15 (1). doi 10.15517/psm.v15i1.26414

Instituto de Nutrición en Centroamérica y Panamá. (2004). La Seguridad Alimentaria y Nutricional: Un Enfoque Integral. Síntesis de los Desafíos y Experiencias en Centroamérica. Recuperado de http://bvssan.incap.int/local/SAN\%20Local/Documentos\%20t\%C3\%A9cnico s/SAN-Un\%20enfoque\%20integral.pdf

Instituto de Nutrición para Centroamérica y Panamá. (2017). Cambio climático y nutrición. Análisis y propuestas metodológicas para evaluar el impacto y respuesta en Centroamérica y República Dominicana. Guatemala: INCAP.

Marín-Arias, L., Ureña Cascante, M. E. y Fernández Rojas, X. (2017). Duración de la lactancia materna exclusiva en una comunidad urbana y otra rural de Costa (c) (i) (2) (2)

La Revista Estudios es editada por la Universidad de Costa Rica y se distribuye bajo una Licencia Creative Commons Atribución-NoComercial-CompartirIgual 3.0 Costa Rica. Para más información envíe un mensaje a revistaestudios.eeg@ucr.ac.cr. 
Dossier: V Simposio Internacional y IX Nacional sobre Derechos de la Niñez y la Adolescencia

Rica. Población y Salud en Mesoamérica, 15(2). doi: 10.15517/psm.v15i2.26408

Martínez Rincón, C. y Rodríguez Cisneros, A. (2002). Influencia de la alimentación en el comportamiento humano a través de la historia. Offarm: Farmacia y Sociedad, 21(7), 80-88.

Ministerio de Salud (2009). Política pública de lactancia materna. Recuperado de: https://www.ministeriodesalud.go.cr/gestores_en_salud/lactancia/politica_la ctancia_materna.pdf

Ministerio de Salud, Instituto Nacional de Estadística y Censos, Fondo de las Naciones Unidas para la Infancia. (2018). Encuesta de Mujeres, Niñez y Adolescencia (EMNA). Informe de resultados de la encuesta. San José, Costa Rica.

Organización Mundial de la Salud. (1998). Promoción de la Salud Glosario. Recuperado de https://apps.who.int/iris/bitstream/handle/10665/67246/WHO_HPR_HEP_98 .1_spa.pdf?sequence=1

Organización Mundial de la Salud. (2003a). Orientaciones estratégicas para mejorar la salud y el desarrollo de los niños y los adolescentes. Recuperado de https://apps.who.int/iris/bitstream/handle/10665/67712/WHO_FCH_CAH_02 .21_spa.pdf?sequence=1

Organización Mundial de la Salud. (2003b). Estrategia mundial para la alimentación del lactante $y$ del niño pequeño. Ginebra: Suiza. Recuperado de https://www.who.int/nutrition/publications/gs_infant_feeding_text_spa.pdf

\section{(C) $(0 \odot$}

La Revista Estudios es editada por la Universidad de Costa Rica y se distribuye bajo una Licencia Creative Commons Atribución-NoComercial-CompartirIgual 3.0 Costa Rica. Para más información envíe un mensaje a revistaestudios.eeg@ucr.ac.cr. 
Dossier: V Simposio Internacional y IX Nacional sobre Derechos de la Niñez y la Adolescencia

Organización Mundial de la Salud (2018). Alimentación del lactante y del niño pequeño. Recuperado https://www.who.int/es/news-room/factsheets/detail/infant-and-young-child-feeding

Organización Mundial de la Salud. (s.f.). Estrategia mundial para la alimentación del lactante $y$ del niño pequeño. Recuperado de https://www.who.int/nutrition/topics/global_strategy_iycf/es/

Organización de Naciones Unidas. (2018). La Agenda 2030 y los Objetivos de Desarrollo Sostenible: una oportunidad para América Latina y el Caribe. Recuperado de https://repositorio.cepal.org/bitstream/handle/11362/40155/24/S1801141_es .$p d f$

Organización de las Naciones Unidas para la Alimentación y la Agricultura, Organización Panamericana de la Salud, Programa Mundial de Alimentos, Fondo de Naciones Unidas para la Infancia. (2018). Panorama de la seguridad alimentaria y nutricional en América Latina y el Caribe 2018. Recuperado de http://www.fao.org/3/CA2127ES/CA2127ES.pdf.

Organización de las Naciones Unidas para la Alimentación y la Agricultura, Fondo Internacional de Desarrollo Agrícola, Fondo de Naciones Unidas para la Infancia, Programa Mundial de Alimentos y Organización Mundial de la Salud. (2019). El estado de la seguridad alimentaria y la nutrición en el mundo 2019. Protegerse frente a la desaceleración y el debilitamiento de la economía. Recuperado de http://www.fao.org/3/ca5162es/ca5162es.pdf

Organización Panamericana de la Salud. (2017). Salud en las Américas, edición del 2017. Resumen: panorama regional y perfiles de país. Washington D.C.: OPS. Recuperado de https://www.paho.org/salud-en-las-americas-2017/wpcontent/ uploads/2017/09/Print-Version-Spanish.pdf 
Dossier: V Simposio Internacional y IX Nacional sobre Derechos de la Niñez y la Adolescencia

United Nations Children's Fund. (2016). From the first hour of life. Making the case for improved infant and young child feeding everywhere. New York: UNICEF. Recuperado de https://www.unicef.org/publications/index_93027.html

Victora, C. G., Bahl, R., Barros, A. J., França, G. V., Horton, S., Krasevec, J., ... y Rollins, N. C. (2016). La lactancia materna en el Siglo XXI: epidemiología, mecanismos y efectos a lo largo de la vida. Serie sobre lactancia materna de The Lancet, 1-4.

World Heath Organization y Wellstar International. (1999). The baby-friendly hospital initiative : monitoring and reassessment : tools to sustain progress. Recuperado de https://apps.who.int/iris/handle/10665/65380 